\title{
Investigation on design method of curved compression surface of inlet on scramjet
}

\author{
Pan $\mathrm{Jin}^{1, *}$, and Jin Feng ${ }^{2}$ \\ ${ }^{1}$ Jiangsu Maritime Institute, Nanjing, China \\ ${ }^{2}$ College of Energy and Power, Nanjing University of Aeronautics and Astronautics, Nanjing, China
}

\begin{abstract}
Suppose that curved compression surface of inlet consists of segments. Two curved surfaces formed by equal compression angles of the micro-element segments and a slight increase in the compression angle of the micro-element segments are designed respectively. The numerical simulation method is used to compare the performance of two curved surfaces with the reference three-wedge compression surface. Select NASA classic test data, in order to determine the turbulence model and calculation method chosen by the numerical simulation Fluent software. The results show: the configuration of the segment compression angle deeply affects compression efficiency of the curved surface compression system. Pressure gradient distribution on the compression surface with constant compression angles segments is nearly constant along the incoming flow direction, and the curved compression surface easily resist the separation of the boundary layer compared to three-wedges compression surface. The approximate calculation method of the bending shock profile is given.
\end{abstract}

Keywords: Scramjet engine, Inlet, Curved compression, Numerical simulation.

\section{Introduction}

Inlet is one of the key components of scramjet. Its performance is tightly associated with the compression efficiency of the compression surface formed the inlet. So, how to design compression surface of inlet is very important. At present, compression ways of conventional inlets are generally axisymmetric compression, two-dimensional plane compression and three-dimensional sidewall compression and so on. ${ }^{[1]-[5]}$

However, traditional compression ways such as wedge compression, conical compression and isentropic compression, still exist various issues. For example, controlling the pressure gradient along the compression surface is very difficult, easily induced boundary layer separation.

In recent years, the compression method of the inlet using a curved surface compression system has been extensively studied. Zhang Kunyuan ${ }^{[6]}$ summarized the surface

\footnotetext{
*Corresponding author: pbrj@163.com
} 
compression technology of hypersonic inlet and proposed the design and reverse design methods of the curved surface compression system.

The curved surface compression system studied in this paper is a curved surface compression system composed of micro element broken lines. It is intended to form a compression system with different compression curvatures by studying the different variation laws of the present microelement fold line compression angle, and investigate the static pressure distribution on the wall surface of the compression system.

\section{Non -viscosity curved compression system}

\subsection{Construction of curved compression surface}

As shown in Figure 1, a two-dimensional curved surface compression system with a total compression angle is divided into $\mathrm{n}$ tiny compression segments. The compression angle of each small segment is a tiny $\Delta \delta_{n}(\mathrm{n}=1,2,3 \ldots)$. The supersonic flow starts from the first limited compression angle and deflects successively along $\mathrm{n}$ tiny compression surfaces. Every deflection of supersonic airflow at a slight angle $\Delta \delta_{n}$ produces a weak compression wave that propagates roughly in the direction of the Mach line. These $\mathrm{n}$ weak compressive waves must in turn intersect the oblique shock waves generated one after another and force the shock waves to bend upward gradually, thus forming this peculiar bending shock wave generated by the curved surface compression system.

Since the flow Mach number $M_{a}$, the initial compression angle $\delta_{0}$, each compression angle $\Delta \delta_{n} \quad(\mathrm{n}=1,2,3 \ldots)$, the total deflection angle of the air flow $\delta\left(\delta>\delta_{0}\right)$, the length of the initial inclined plate $L_{0}$ and the length of the curved surface $L$ are used as design parameters, compile the design program of curved surface compression. Through the calculation of the program, it is possible to construct curved compression profiles with different compression effects. It can be seen from Figure 1 that this concave curved compression surface is composed of a section of inclined wedge of initial length $L_{0}$, followed by a section of single wedge with angular deflection $\delta_{0}$, followed by a series of tiny broken lines with $\mathrm{n}$ sections and angular deflection $\Delta \delta_{n}$.

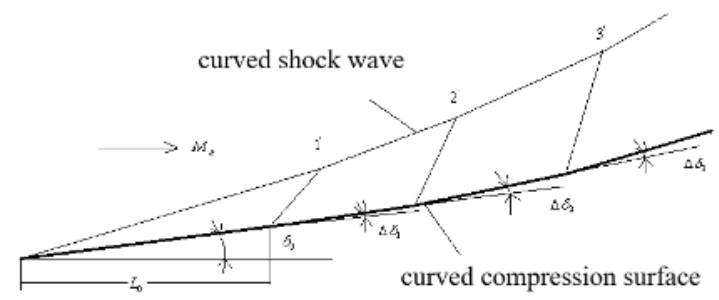

Fig. 1. Sketch of the structure of curved surface compression system.

\subsection{Bending shock wave flow field}

By changing the above design parameters, a series of bending shock wave compression fields with different characteristics can be obtained. The constructed curved surface compression surface non- Viscosity flow field numerical was carried out at $M a=5$ by using Fluent software . Figure 2 is its Mach number contour and static pressure contour. It can be clearly seen from the figure that the first oblique shock wave and the subsequent 
weak compression wave intersect in order to form a shock wave that gradually bends upward

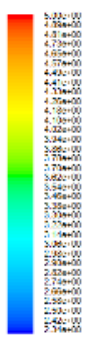

(a) Mach number contour

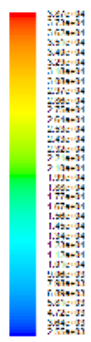

(b) static pressure contour

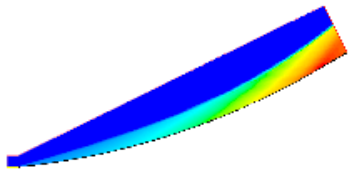

Fig. 2. Flow field diagram of curved shock surface compression system.

\section{Approximate calculation of bending shock wave profile}

Suppose that given a curved compression surface capable of generating a curved shock wave, as shown in Figure 3. On the compression surface, discrete points with small intervals are sequentially taken out, and the continuous curved surface can be regarded as a straight wall of micro elements connected by these points. When the supersonic flow flows through the curved surface, since the compression process is very weak, it can be approximated that each point will emit an isentropic compression wave. By calculating the unit of the intersection of the same family wave on the two adjacent isentropic compression waves, the flow parameters along the wall, the coordinates of the points on the shock wave and the post-wave parameters can be obtained.

Since these micro-segments can be made infinitely small, the effect of the slip surface generated by the intersection of the two waves and the reflected wave on the downstream flow field can be ignored in the calculation. If these micro-segments are of finite length, then this processing method is a certain approximation.

Figure 3 shows a unit process of bending shock wave formation. It can be seen from the figure that when the supersonic airflow flows through a known two-dimensional curved surface, this curved surface can be decomposed into $\mathrm{n}$ micro-segments, and $\mathrm{n}$ straight-wall micro- Segment replaces this continuously changing concave surface. Of course, in practical applications, limited by the manufacturing process and structural strength, the designed two-dimensional curved compression surface should have a certain initial compression angle. At this time, the first point of the two-dimensional curved surface produces a shock wave, and the starting point of the shock wave is the starting point of the curved compression surface. 


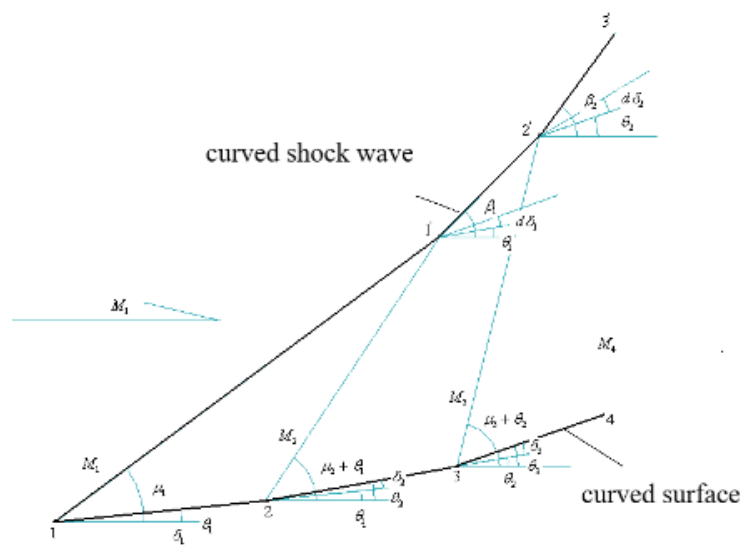

Fig. 3. Schematic diagram of approximate calculation of bending shock wave.

If the coordinates of points on the surface are known as $\left(x_{1}, y_{1}\right),\left(x_{2}, y_{2}\right), \cdots,\left(x_{n}, y_{n}\right)$, you can get the slope of each segment of the surface $\Delta y / \Delta x$, angle relative to incoming flow $\theta$ and Chamfer (the difference between adjacent straight line angles) $\delta$.

Assume the coordinates of the points on the shock surface $\operatorname{are}\left(x_{1}^{\prime}, y_{1}^{\prime}\right),\left(x_{2}^{\prime}, y_{2}^{\prime}\right), \cdots,\left(x_{n}^{\prime}, y_{n}^{\prime}\right)$ and known Mach number $M_{1}, P_{1}, P_{t 1}, T_{1}, \rho_{1}$.

The specific steps of approximate calculation are as follows:

As shown in Figure 3, the coordinates of the intersection point 1' are determined by the coordinates of 1,2 points.

According to $M_{1}, \delta_{1}$ and formula (1), (2), we can find $M_{2}$, then solve the following system of equations(3) .

$$
\begin{gathered}
\frac{1}{\operatorname{tg} \delta}=\left(\frac{\gamma+1}{2} \frac{M_{1}^{2}}{M_{1}^{2} \sin ^{2} \beta-1}-1\right) \operatorname{tg} \beta \\
M_{2}^{2} \sin ^{2}(\beta-\delta)=\frac{1+\frac{\gamma-1}{2} M_{1}^{2} \sin ^{2} \beta}{\gamma M_{1}^{2} \sin ^{2} \beta-\frac{\gamma-1}{2}} \\
\left\{\begin{array}{l}
y_{1}^{\prime}-y_{1}=\left(x_{1}^{\prime}-x_{1}\right) \tan \left(\mu_{1}\right) \\
y_{1}^{\prime}-y_{2}=\left(x_{1}^{\prime}-x_{2}\right) \tan \left(\mu_{2}+\theta_{1}\right)
\end{array}\right.
\end{gathered}
$$

Then get $\left(x_{1}^{\prime}, y_{1}^{\prime}\right), \beta_{1}, \mu_{2}$ corresponds to the shock angle and the Mach angle of $M_{2}$. By repeating the above calculation process, we can get the coordinates of other points on the shock profile and generate the shock profile. This approximate calculation method provides great convenience for the subsequent 2-d inlet design, which requires the compression surface shock to converge at the lip and determine the lip position at the design point.

\section{Calculation of viscous bending shock wave flow field}

In order to explore the possibility of applying this new type of compression system to supersonic/hypersonic inlets, the following will use the hypersonic two-dimensional inlet as 
a research platform to try to compare this curved compression surface with the plane inclined wedge compression.

\subsection{Calculation method}

Under the condition of super/supersonic flow, we use Fluent software to perform viscosity numerical simulation. The specific calculation method is as follow:

The calculation process uses the coupled compressible N-S equation and the model transport equation to solve implicitly. The molecular viscosity coefficient is calculated using Sutherland formula. Standard wall function is for near wall processing. Proportional encrypted mesh with attached layer near the wall of the calculation model. Specific heat ratio $\gamma=1.4$. Boundary conditions of pressure far-field, pressure outlet and non-slip adiabatic solid wall are used in the calculation. The calculation convergence condition is that the residual of each index drops to the order of $10^{-3}$ and the inlet and outlet flow balance. In this paper, Fluent software numerical simulation used turbulence models is $k-\varepsilon$ model.

\subsection{Physical models}

Each compression angle and each increment of compression angle of the unconventional curved surface compression system designed in this paper are respectively $\Delta \delta=1^{0}$ and $d \delta=0.15^{\circ}$. The compression angles of the three wedge surfaces of the reference wedge compression two-dimensional inlet are respectively $6^{\circ}, 6.5^{\circ}$ and $7.5^{\circ}$. The total deflection angle is also $20^{\circ}$. Select the design point Mach number $M a=5 \cdot 3^{[7]}$.

The calculation conditions are incoming flow Mach number 5.3, static pressure $2250 \mathrm{~Pa}$, and static temperature $221.6 \mathrm{~K}$. Using Fluent software, numerically simulate the above three two-dimensional compression surfaces, and analyze their flow field under uniform inflow conditions.

\subsection{Results and discussion}

Fig.4 is the static pressure distribution on the compression surface of these three two-dimensional surfaces under the condition of uniform incoming flow. It can be seen from the figure that the static pressure on the compression surface of the two unconventional curved compressing surfaces rises slowly along the flow direction, and are approximately equal pressure gradient. The three-wedge compression is completed by the three-wedge compression of the air flow. Therefore, the pressure rise on the compression surface is achieved through three pressure jumps. We can also see from the figure that the compression surface with unconventional curved surface have shorter length. 


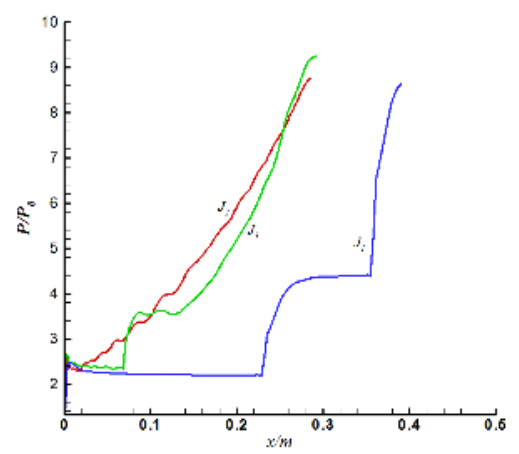

Fig. 4. Ma 5.3 static pressure distribution on the compression surfaces.

According to the following empirical formula ${ }^{[8]}$ for predicting turbulent boundary layer separation:

$$
\frac{P_{b}}{P_{s}}=1+0.2 \frac{\gamma M a_{s}^{2}}{\left(M a_{s}^{2}-1\right)^{0.25}}
$$

In the formula: $P_{b} / P_{S}$-----Shock wave critical pressure ratio leading to turbulent boundary layer separation.

$$
M a_{s} \text {----- Local Mach number of airflow before separation. }
$$

The initial compression angle of the conventional three-wedge compression is $6^{0}$, and the initial compression angle of the unconventional curved surface compression is also $6^{0}$. According to formula (4), it can be calculated that the pressure ratio of the incoming Mach number $=5.3$ after the $6^{0}$ oblique wedge compression is 4.63 . The critical pressure ratio leading to separation of the boundary layer here is $P_{b} / P_{s}=3.82$. The compression angle of the second wedge of the conventional three-wedge compression is $6.5^{\circ}$, the pressure ratio of the second shock wave is 2.054 . For unconventional compression surfaces, the initial wedge shock wave is followed by a compression wave, weaker intensity than shock wave compression. The pressure ratio calculated by numerical simulation is only 1.134 , which is far lower than the critical pressure ratio for separation of the boundary layer. Obviously, in the integrated design of hypersonic aircraft, the inlet with unconventional compression profile has great advantages in maintaining the stability of the boundary layer on the compression surface.

In the design of the conventional three-wedge compression two-dimensional inlet, in order to shorten the length of the inlet, a diagonal wedge with a large compression angle is used, and the shock wave is used to compress the air flow, which may cause separation occurs on the boundary layer.

\section{Conclusion}

In this paper, design method of forming a curved surface compression system using segments is proposed. The following research results were obtained:

(1) By reasonably configuring the segment compression angles that make up the surface compression system, a surface compression system with different compression efficiency is formed. Under the condition of supersonic incoming, combined curved surface compression system carefully can form ideal curved shock waves.

(2) Analyzing the flow field of curved surface compression system composed of segments and using shock wave and isentropic compression theory, can be approximated to 
obtain the curved shock wave profile, which provides convenience for the design of curved surface compression inlet.

(3) Under the condition of supersonic incoming flow, the static pressure distribution on the compression surface with constant compression angles segments is nearly a diagonal line along the incoming flow direction. It can be seen that by configuring the compression angle of segments forming the compression system of the curved shock curved surface, it is possible to control the law of the pressure rise of the compression surface and avoid the separation of the boundary layer.

The authors thank the China National Fund Committee for its financial support. The work was financed by national natural science funds 90916029 .

\section{References}

1. Rausch D R, McClinton C R. Hyper-X Program Overview [C]. Proceedings of 14th International Symposium on Air Breathing Engines. ISABE Paper 99-7213.

2. Leonard E.Stitt, Donald L. Chubb, “ Investigation Of Three Mach 7 External-Compression Axisymmetric Inlets From Mach 1.9 To 6.8," NASA TM X-413, 1960

3. McClinton C R, Rausch D R. Hyper-X Program Status. AIAA 2001-1910.

4. J. Bradford, J.Olds, R.Bechtel,T.Cormier,etc. "Exploration of the Design Space for the ABLV-GT SSTO Reusable Launch Vehicle," AIAA 2000-5136

5. Scott A Berry, Aaron H Auslender and Authur D Dilley. Hypersonic boundary-layer trip development for Hyper-X. AIAA 2000-4012.

6. Zhang Kunyuan. Summarization of hypersonic air inlet curved surface compression technology [J]. Propulsion Technology, 2018, 39(10): 2227-2235.

7. Pan J, Zhang K Y. Experimental and Numerical Investigation of a Curved Compression System Designed on Constant Pressure Gradient [R]. AIAA 2009-5270.

8. Liu Guoqiu, "Principle of Integrated Rocket Engine", Beijing, Aerospace Publishing Press, 1993. 